\title{
PARTIAL FFT DEMODULATION: A DETECTION METHOD FOR DOPPLER DISTORTED OFDM SYSTEMS
}

\author{
Srinivas Yerramalli, Milica Stojanovic and Urbashi Mitra \\ University of Southern California, North Eastern University, University of Southern California
Los Angeles CA
Boston MA
}

\begin{abstract}
In mobile OFDM systems, such as underwater acoustic and digital video broadcasting (DVB) systems, time variation causes severe inter-carrier interference (ICI) which limits data detection performance. To address this problem, a signal processing strategy using several partial-interval FFTs instead of a conventional full-interval single FFT is proposed. Weighted combining of the partial FFT outputs coupled with standard OFDM processing, allows high performance symbol-by-symbol detection even in highly time-varying channels. Numerical examples show performance gains of several $\mathrm{dB}$ over a conventional receiver, thus indicating that an order of magnitude reduction in BER is achievable at a minimal increase in complexity.
\end{abstract}

\section{INTRODUCTION AND PROBLEM MOTIVATION}

Orthogonal Frequency Division Multiplexing (OFDM) has emerged as an attractive signaling scheme for modern wireless wideband communications such as 3GPP LTE, WIMAX, Wireless LANs, Digital TV Broadcasting (DVB), Vehicle to Vehicle (V2V) communications, and has recently been considered for underwater acoustic (UWA) communications. The latter are of special interest as they exemplify severely Doppler distorted channels, with Doppler factors as high as $10^{-4}$ (normalized residual Doppler in excess of $10 \%$ ).

Interest in OFDM stems from the fact that it decomposes a timeinvariant frequency selective channel into a set of independent subchannels that are free of inter-symbol interference. However, OFDM is highly sensitive to temporal channel variation, frequency offsets and motion-induced Doppler distortion, which destroy carrier orthogonality and introduce inter-carrier interference (ICI). For fast moving systems, the channel is highly time-varying, and the resulting ICI severely degrades the performance of conventional data detection schemes which do not explicitly mitigate ICI.

Most approaches to ICI mitigation can be classified into two categories: pre-FFT and post-FFT processing. Pre-FFT linear processing schemes such as time-domain windowing to shorten the ICI are considered in [1], while time-varying filters have been designed in [2] to enable per-tone equalization. Post-FFT processing schemes have received special attention as they permit low complexity receiver implementations. In [3-5] (and the references therein), several post-FFT decision feedback equalization schemes with time and frequency domain Minimum Mean Squared Error (MMSE) receivers have been proposed. Low complexity data detection algorithms that assume linearly varying tap gains have been proposed for wireless

This research has been funded in part by the following grants and organizations: ONR MURI grant N00014-07-1-0738, ONR N00014-09-1-0700, NSF CNS-0832186, NSF CNS-0821750 (MRI), NSF OCE-0520324 and the University of Southern California's Provost Office. radio (see [6,7] and the references therein). In wideband OFDM such as UWA and Ultra Wide Band (UWB) OFDM systems [8], Doppler distortion causes time-scaling of the signal and as such may not be accurately modeled using linearly varying tap-gains. In [9], a low complexity detector for OFDM in time-varying underwater acoustic communications which models time-scaling as a phase shift has been proposed, and in [8] a synchronization algorithm to jointly estimate the frequency offset and the Doppler-scaling factor is presented. An adaptive algorithm for estimating an underwater channel is derived in [10] and the ICI is compensated using a MMSE equalizer.

In this paper, we propose a signal processing strategy to combat ICI in OFDM systems. We target the source of ICI - the channel's time-variation - by performing several partial-interval FFTs instead of the conventional, full-interval single FFT. Assuming that the channel is fixed over the duration of each partial FFT interval (but possibly varying from one such interval to another), FFT outputs are weighted before combining to suppress the ICI. After combining, the signal is subject to conventional OFDM detection. The detector may also incorporate additional, explicit ICI cancellation, but our results indicate that partial FFT demodulation suppress ICI sufficiently enough that further cancelation is not necessary in many cases of practical interest. Though the idea of dividing the OFDM interval into several blocks has been investigated earlier (e.g. see [11]), the novelty of our approach is in the combining algorithm and the low cost of implementation.

Weighted combining of the partial FFT segments effectively acts as front-end filtering, but unlike general filtering, it requires only a minor increase in computational complexity (several additional FFTs). Moreover, the method does not rely on any prior knowledge of the channel or the channel statistics, and can thus be used to compensate for diverse sources of ICI.

Using a simple example we first demonstrate the performance improvement that can be obtained by partial FFT demodulation for a known channel. We then propose an algorithm for computing the combiner weights without the a priori knowledge of the channel variation or the Doppler distortion. Finally, we couple the partial FFT combiner with a standard OFDM detection method that incorporates frequency-domain channel estimation. The algorithm performance is assessed through simulation, using examples of a wideband underwater acoustic system and a DVB system. The results indicate that a significant performance improvement can be obtained by using only a few partial FFTs.

The rest of this paper is organized as follows. Section 2 introduces the concept of partial FFT demodulation. Section 3 outlines the complete receiver algorithm that incorporates recursive computation (across carriers) of the combiner weights and the channel. Simulation results are presented in Section 4, while Section 5 concludes the paper. 


\section{SYSTEM MODEL AND KEY IDEA}

\subsection{Signal Model}

We consider an OFDM system with $K$ carriers. Each element of the vector $\mathbf{d}=\left[d_{1}, d_{2}, \cdots, d_{K}\right]$ of information symbols is modulated onto one of the OFDM subcarriers. The transmitted symbols are assumed to be drawn from a finite constellation, such as 4-QAM, which we consider for illustration. Let $T$ and $T_{g}$ denote the duration of the OFDM symbol and the cyclic prefix, respectively. The first carrier is at a frequency $f_{0}$, and the subcarrier spacing is $\Delta f=1 / T$. The $k^{\text {th }}$ subcarrier frequency is $f_{k}=f_{0}+(k-1) \Delta f$, and the total bandwidth is $B=K \Delta f$. The transmitted OFDM signal in passband is given by

$$
s(t)=\operatorname{Re}\left\{\sum_{l=1}^{K} d_{l} e^{j 2 \pi f_{l} t}\right\}, t \in\left[-T_{g}, T\right] .
$$

A generic model for the time-varying channel can be expressed as

$$
h(\tau, t)=\sum_{p=1}^{P} h_{p}(t) \delta\left(\tau-\tau_{p}(t)\right)
$$

where $h_{p}(t)$ and $\tau_{p}(t)$ are the time-varying gain and delay of the $p^{t h}$ path. For wireless systems, the path gains are time-varying, but the delays are assumed to be time-invariant, i.e., $\tau_{p}(t)=\tau_{p}$. However, in highly mobile systems such as UWA, the delays can be modeled as linearly varying with time, $\tau_{p}(t)=\tau_{p}-a t$, where $a$ is the timescaling Doppler factor.

\subsection{Partial FFT Demodulation}

Assuming the generic channel model the signal at the receiver can be expressed as

$$
\begin{aligned}
r(t) & =\operatorname{Re}\left\{\sum_{p=1}^{P} h_{p}(t) s\left(t-\tau_{p}(t)\right)\right\}+n(t) \\
& =\operatorname{Re}\left\{\sum_{p=1}^{P} \sum_{l=1}^{K} h_{p}(t) d_{l} e^{j 2 \pi f_{l}\left(t-\tau_{p}(t)\right)}\right\}+n(t) .
\end{aligned}
$$

We assume that the timing synchronization is precise. After removal of the cyclic prefix, the OFDM block interval of duration $T$ is divided into $M$ non-overlapping intervals, and each is assigned to one partial FFT demodulator. The output of the $m^{t h}$ partial demodulator for the $k^{\text {th }}$ OFDM subcarrier is given by ${ }^{1}$

$$
\begin{aligned}
y_{k}(m)= & \frac{1}{T} \int_{\frac{(m-1) T}{M}}^{\frac{m T}{M}} r(t) e^{-j 2 \pi f_{k} t} d t \\
= & \sum_{p} \sum_{l} d_{l} \int_{\frac{(m-1) T}{M}}^{\frac{m T}{M}} h_{p}(t) e^{-j 2 \pi f_{l} \tau_{p}(t)} e^{j 2 \pi\left(f_{l}-f_{k}\right) t} d t \\
& +n_{k}(m), m=1,2, \cdots, M .
\end{aligned}
$$

The noise terms $n_{k}(m)$ are independent for a fixed subcarrier $k$ and varying $m$, but are correlated for a fixed $m$ and varying $k$.

If the path gains and the delays are slowly varying over the in-

\footnotetext{
${ }^{1}$ We use the continuous-time notation for simplicity of illustration. In practice, the system operates on sampled signals and the integrals are efficiently computed using the FFT algorithm.
}

terval $T / M$, The received signal can then be approximated as

$$
\begin{aligned}
y_{k}(m) & \approx \sum_{p=1}^{P} \sum_{l=1}^{K} d_{l} h_{p}(m) e^{-j 2 \pi f_{l} \tau_{p}(m)} I_{l-k}(m)+n_{k}(m) \\
& =\sum_{l=1}^{K} d_{l} H_{l}(m) I_{l-k}(m)+n_{k}(m),
\end{aligned}
$$

where $h_{p}(m)$ and $\tau_{p}(m)$ are the relevant mid-point values of the path gains and delays. The effective channel gain as seen by the $k^{t h}$ subcarrier in the $m$-th demodulation interval $[(m-1) T / M, m T / M]$ can then be expressed as

$$
H_{k}(m)=\sum_{p=1}^{P} h_{p}(m) e^{-j 2 \pi f_{k} \tau_{p}(m)}
$$

The function $I_{i}(m)$ describes the effect of partial integration and is given by

$$
I_{i}(m)=\int_{\frac{(m-1) T}{M}}^{\frac{m T}{M}} e^{j 2 \pi i \Delta f t} d t, i=-(K-1), \ldots(K-1) .
$$

We note that $\sum_{m} I_{i}(m)=\delta_{i}$.

For the special case of a channel with linearly varying path delays, the time-varying frequency response can be expressed as

$$
H_{k}(m)=e^{j 2 \pi f_{k} a \frac{(2 m-1) T}{2 M}}\left[\sum_{p} h_{p}(m) e^{-j 2 \pi f_{k} \tau_{p}}\right],
$$

where $a$ is the Doppler scaling factor.

\subsection{Combining Partial FFT Outputs}

If the partial segments are simply added, the result is that of full FFT demodulation. However, if the segments are weighted before combining, a judicious selection of weights allows one to partially compensate for the time-variation in the channel response. The weighted combining is performed as follows.

Let us define $\mathbf{y}_{k}=\left[y_{k}(1), y_{k}(2), \cdots, y_{k}(M)\right]^{T}$ as the vector of partial FFT segments for the $k^{t h}$ subcarrier, and let $\mathbf{p}_{k}=$ $\left[p_{k}(1), p_{k}(2), \cdots, p_{k}(M)\right]^{T}$ be the vector of the corresponding weights. The combining then yields a signal

$$
x_{k}=\mathbf{p}_{k}^{H} \mathbf{y}_{k} \text {. }
$$

As mentioned earlier, if $\mathbf{p}_{k}=[1,1, \cdots, 1]^{T}, \forall k$, the situation is equivalent to performing a single, full-interval FFT. For the ideal case of a time-invariant channel, we have that

$$
x_{k}=H_{k} d_{k}+n_{k},
$$

and symbol-by-symbol detection is optimal. When the channel is time-varying, we will tailor the combiner weights to achieve the desired output in this form.

It is important to note that an appropriate choice of the vectors $\mathbf{p}_{k}$ allows one to compensate for the time-variation of the channel to some degree, thus reducing the ICI but not eliminating it. Even if one were to implement optimal front-end filtering, i.e. a matched filter for each subcarrier, the resulting output would contain ICI. Partial FFT demodulation followed by combining mimics the operation of front-end filtering, and, hence, cannot completely eliminate the ICI. However, it can significantly reduce the effect of ICI as compared to 
the conventional FFT demodulation, and it is also much simpler to implement than pre-FFT filtering. It thus enables a low complexity implementation, where only a few additional FFTs are required at the receiver front-end. The signal after combining can be processed in a standard manner, without dedicated ICI suppression such as banded equalization [12]. Clearly, partial FFT demodulation can also be combined with ICI equalization; however, our goal at the moment is to investigate its performance as a stand-alone alternative.

\subsection{Illustrative Example}

To illustrate the performance improvement that can be obtained by using the proposed technique, we consider a single-path, unit gain channel, where the only distortion is due to Doppler scaling. Denoting by $a$ the Doppler scaling coefficient, the received signal without noise can be expressed as

$$
r(t)=\operatorname{Re}\left\{\sum_{l=1}^{K} d_{l} e^{j 2 \pi f_{l} t(1+a)}\right\},
$$

and the partial FFT demodulation yields

$$
y_{k}(m) \approx \sum_{l=1}^{K} d_{l} e^{j 2 \pi f_{l} a \frac{(2 m-1) T}{2 M}} I_{l-k}(m) .
$$

Assuming that the Doppler scaling coefficient $a$ is known at the receiver, the effect of weighted combining is to de-rotate the FFT outputs so as to properly align them, resulting in

$$
x_{k}=\sum_{m=1}^{M} e^{-j 2 \pi f_{k} a \frac{(2 m-1) T}{2 M}} y_{k}(m)=d_{k}+\xi_{k},
$$

where $\xi_{k}$ is the residual interference on the $k^{t h}$ carrier.

Fig. 1 shows the scatter plots of this signal for several values of $M$ when 4-QAM is used. We assume system parameters representative of an underwater system. At $a=10^{-4}$, the normalized residual Doppler shift $a f_{k} / \Delta f$ ranges from $20 \%$ for the lowest subcarrier frequency to $30 \%$ for the highest. Shown in the top left corner is the scatter plot of conventional FFT output $y_{k}$. The remaining three plots show the scatter plot of the combined partial FFT demodulator outputs $x_{k}$ for $M=2,4,8$, Clearly, phase rotation alone severely impacts the performance of conventional detection, while partial FFT combining significantly reduces ICI.

\section{PRACTICAL ESTIMATION ALGORITHMS}

The weighing coefficients $\mathbf{p}_{k}$ in the previous example were determined based on the a priori knowledge of the Doppler distortion parameter. However, in practical receivers, prior knowledge of the channel distortion is not available, and the weights need to be estimated. In this section, we present an adaptive algorithm that computes the combiner weights recursively across subcarriers. The algorithm is based on the assumption that the combiner weights $\mathbf{p}_{k}$ and channel frequency response $H_{k}$ as defined by the expressions $(7,8)$ are slowly varying with the subcarrier index $k$.

The algorithm operates in several steps. In the first step, combining is performed to yield the signal $x_{k}=\mathbf{p}_{k}^{H} \mathbf{y}_{k}$. Next, using the estimate of the channel frequency response on the previous carrier, $\hat{H}_{k-1}$, the signal $x_{k}$ is equalized to form the estimate of the data
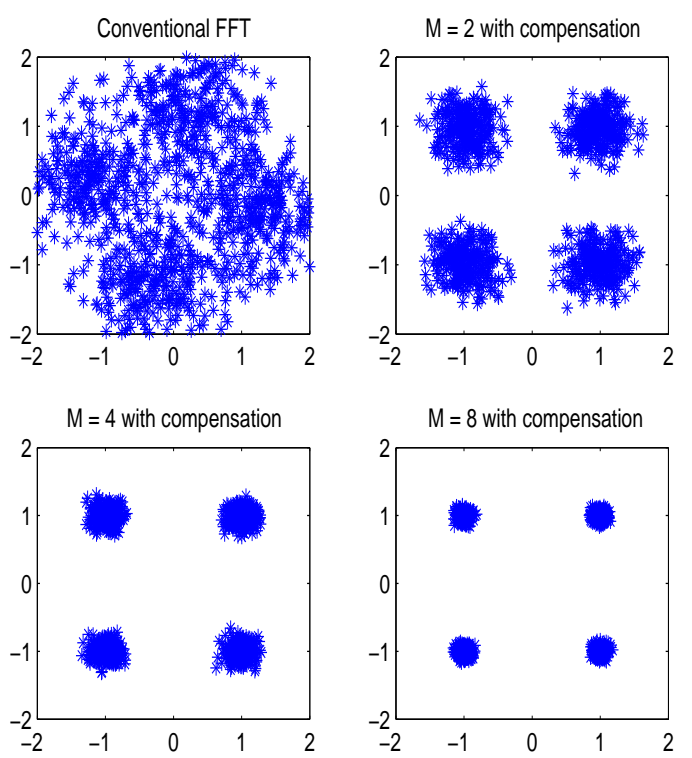

Fig. 1. Scatter plots after conventional FFT demodulation and after partial FFT demodulation and combining (11). Single-path channel with Doppler scaling coefficient $a=10^{-4} ; 1024$-carrier OFDM in the $24 \mathrm{kHz}-36 \mathrm{kHz}$ frequency band.

symbol $\hat{d}_{k}$, based on which the symbol decision $\tilde{d}_{k}$ is made:

$$
\hat{d}_{k}=\frac{x_{k}}{\hat{H}_{k-1}} ; \tilde{d}_{k}=\operatorname{dec}\left(\hat{d}_{k}\right)
$$

The channel frequency response for the current carrier is then computed as

$$
\hat{H}_{k}=\eta \hat{H}_{k-1}+(1-\eta) \frac{x_{k}}{\hat{d}_{k}}
$$

where $0<\eta<1$ is the channel update parameter. This is a simple channel estimator, but one that suffices to illustrate the receiver operation. More sophisticated channel estimation algorithms can of course be employed.

Assuming correct symbol decisions, or using pilot symbols where available, the error at the combiner output is evaluated as

$$
e_{k}=\hat{H}_{k} \tilde{d}_{k}-x_{k}
$$

This error is used to drive an adaptive algorithm for the combiner weights, for example the Recursive Least Squares (RLS) algorithm: $\mathbf{p}_{k+1}=\mathbf{p}_{k}+\operatorname{RLS}\left[\mathbf{y}_{k}, e_{k}\right]$. The complete algorithm is summarized below.

The parameter $\eta$ controls the channel estimate, while the parameter $\lambda$ controls the combiner weights. These parameters can be varied to suit the particular channel and the signal to noise ratio (SNR) at hand. In general, for a highly frequency-selective channel, the rate of change of $H_{k}$ is expected to be (much) greater than the rate of change of $\mathbf{p}_{k}$. The nominal RLS convergence time, $2 M$, determines the minimum number of pilots. The computational cost of the RLS algorithm is $O\left(M^{2}\right)$ and the FFTs $\operatorname{cost} O\left(M N_{s} \log N_{s}\right)$, where $N_{s}$ is the size of the each FFT performed at the receiver.

Pilot symbols $\mathcal{P}$ and are used to periodically train the receiver. Pilot insertion beyond those needed for initial RLS convergence is 


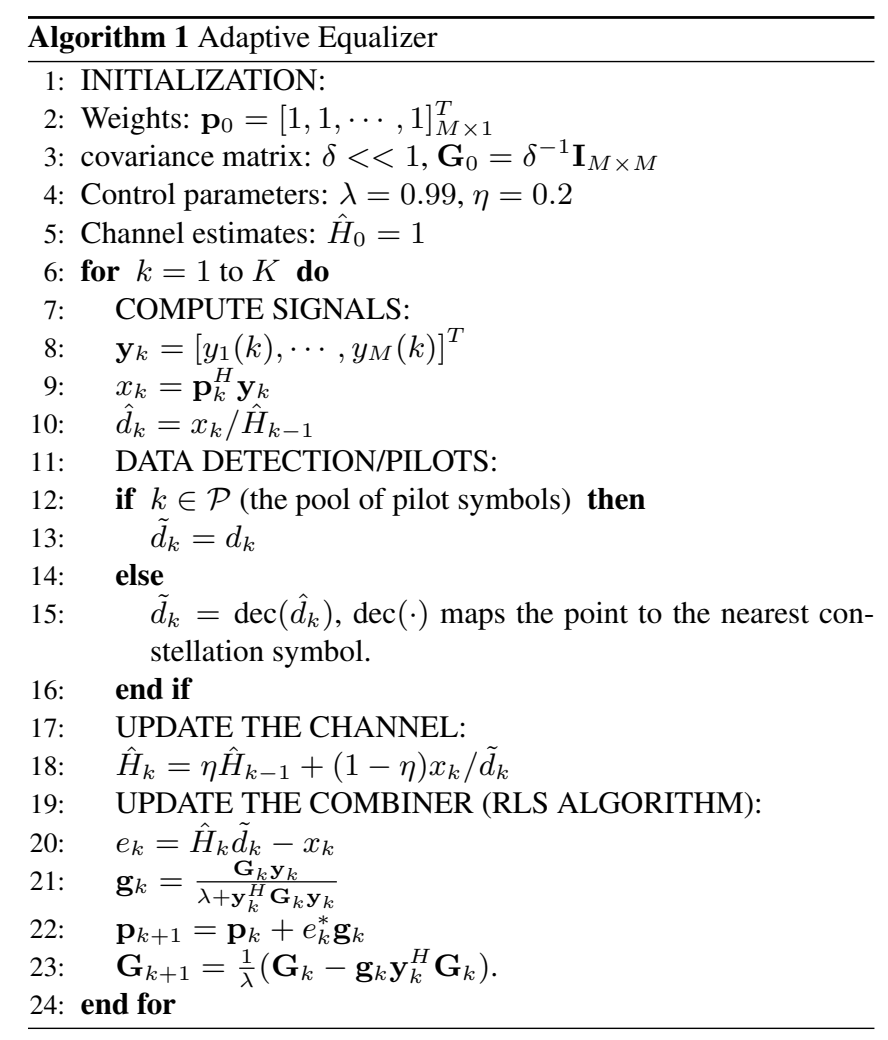

not necessary for mildly distorted channels, but it becomes necessary for channels that exhibit spectral nulls. On such channels, a subcarrier that is near a deep fade is likely to cause a symbol error, which will propagate. To prevent error propagation, pilot symbols can be organized in groups which are periodically inserted into the data stream. In addition, error control coding with interleaving in frequency, as well as multiple receive elements which are normally used; will help in these situations.

\section{SIMULATION RESULTS AND DISCUSSION}

In this section, we present simulation results to demonstrate the performance of partial FFT demodulation. We consider two example systems: an underwater acoustic OFDM system, and a high speed wireless vehicle-to-vehicle system. In each case, we consider an uncoded system having three receive elements with maximal ratio combining.

\subsection{Underwater Acoustic OFDM System}

We consider a shallow water communication link operating in the $24 \mathrm{kHz}-36 \mathrm{kHz}$ acoustic frequency band. The nominal path gains, calculated for an example system geometry, are shown in Fig. 2. Time variation is assumed to occur due to motion. The ratio of the relative transmitter/receiver velocity $v$ to the speed of sound $c=1500$ $\mathrm{m} / \mathrm{s}$ can reach values in excess of $10^{-3}$ in an underwater system, and to account for such situations, the received signal is normally resampled using a coarse estimate of the ratio $v / c$. However, since the time scaling is large to begin with, even a small error results in a significant residual time scaling. For the present example, we consider the residual Doppler scaling factor $a=10^{-4}$. Note that

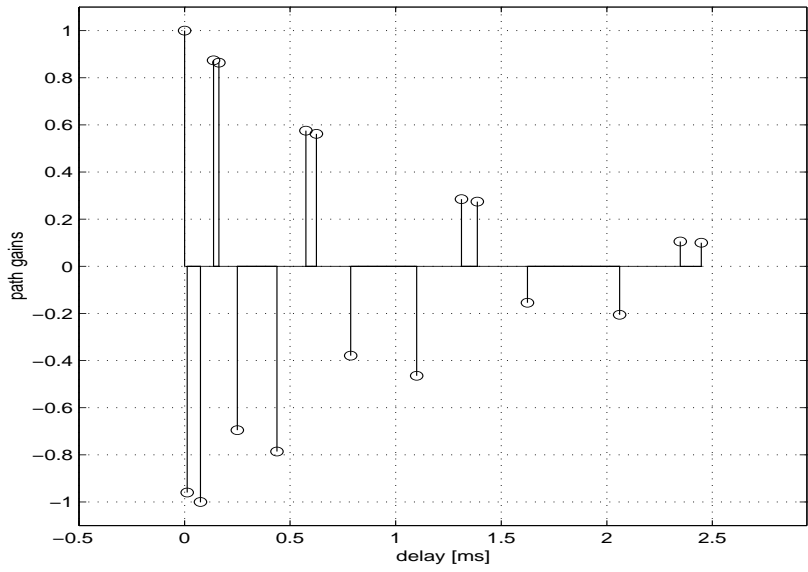

Fig. 2. Path gains for the underwater acoustic test channel are calculated for a $2 \mathrm{~km}$ distance, $15 \mathrm{~m}$ water depth, transmitter and receiver in mid water over soft bottom, spreading factor of 1.5 and the center frequency of $31 \mathrm{kHz}$.

such a value may result even in a system where the transmitter and receiver are not moving intentionally, but only drifting with currents.

Fig. 3 shows the symbol error rate (SER) as a function of SNR, using a 1024-carrier OFDM system. For full-interval demodulation, i.e. $M=1$, the SER is very high, and in most scenarios the detector fails even when additional forward error correction is used. In contrast, partial FFT demodulation reduces the SER by more than an order of magnitude. This is due to the fact that demodulator outputs are properly weighted before combining, thus compensating for the ICI and enabling subsequent channel estimation and data detection. As the number of partial FFT outputs $M$ increases, the number of combiner weights to be recursively estimated also increases and results in a performance tradeoff due to increasing estimation noise. For the current example, the performance observed with $M=8$ is approximately the same as with $M=4$. The effect of diminishing returns observed with a further increase in $M$ can also be explained by the fact that partial FFT combining accounts only for the frontend filtering, and not for complete removal of ICI. The error floor thus remains; however, as Fig. 3 demonstrates, it is significantly reduced as compared to that observed with conventional detection.

\subsection{High speed wireless systems}

We consider a DVB system using $K=8192$ carriers with a bandwidth $B=8 \mathrm{MHz}$ centered at $800 \mathrm{MHz}$. The channel is modeled using six independent Rayleigh fading paths, each with a Jakes' spectrum specified by a normalized Doppler spread of $f_{d} T=0.2$, and a delay spread of $6 \mu s$ [13].

Fig. 4 shows the resulting SER. The performance improvement is not as striking as for the UWA channel case, but nonetheless, it exists. Notably in the higher SNR region, an improvement of $2 \mathrm{~dB}$ is gained by using just two FFTs. The improvement saturates with a further increase in $M$ as sufficient resolution is reached, i.e., there is nothing more to be gained by further splitting the FFT demodulation interval at this value of Doppler spread. 


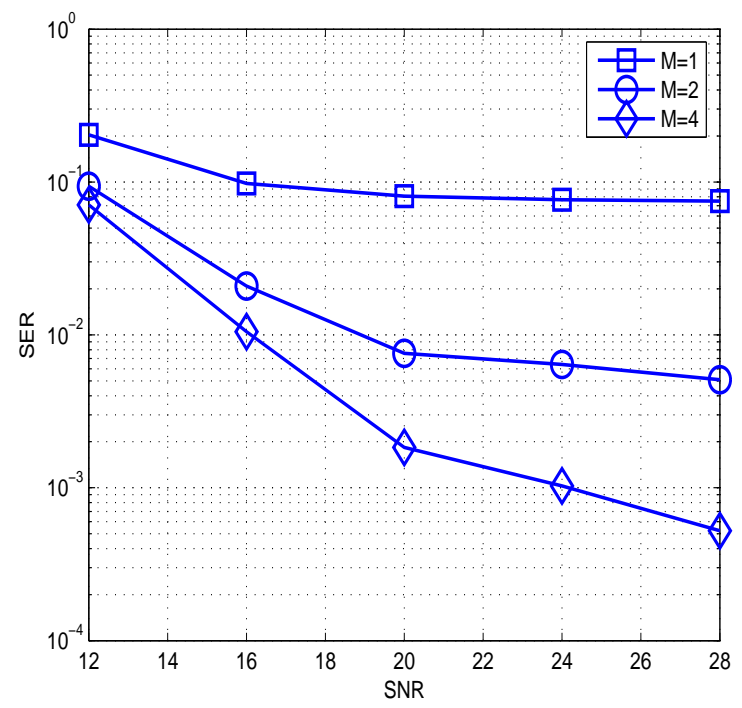

Fig. 3. Symbol error rate as a function of SNR for an underwater acoustic test channel with Doppler scaling factor $a=10^{-4}$ : conventional system $(M=1)$, and partial FFT demodulation with varying number of intervals $M$.

\section{CONCLUSIONS}

In this paper, we considered an OFDM system in a fast varying mobile scenario with severe inter-carrier interference. To counteract this effect, FFT demodulation was considered over partial segments, such that each segment can be appropriately weighted before combining. The resulting signal is processed by a conventional OFDM detector that employs an adaptive channel estimator. An algorithm for recursively determining the combiner weights is proposed and the performance improvement obtained by using partial FFTs is demonstrated for underwater acoustic communications and DVB applications.

\section{REFERENCES}

[1] P. Schniter, "Low complexity equalization of OFDM in doubly selective channels," IEEE Transactions on Signal Processing, vol. 52, no. 4, Apr 2004.

[2] I. Barhumi, G. Leus, and M. Moonen, "Equalization for OFDM over doubly selective channels," IEEE Transactions on Signal Processing, vol. 54, no. 4, Apr 2006.

[3] X. Cai and G. Giannakis, "Bounding performance and suppressing intercarrier interference in wireless mobile OFDM," Communications, IEEE Transactions on, vol. 51, no. 12, pp. 2047-2056, Dec. 2003.

[4] K. Fang, L. Rugini, and G. Leus, "Low complexity block Turbo equalization for OFDM systems in time varying channels," IEEE Transactions on Signal Processing, vol. 56, no. 11, pp. 5555-5566, Nov 2008.

[5] I. Barhumi, G. Leus, and M. Moonen, "Time and frequency domain per-tone equalization for OFDM in doubly selective channels," Elsevier Journal on Signal Processing, vol. 84, no. 11, pp. 2055-2066, Nov 2004.

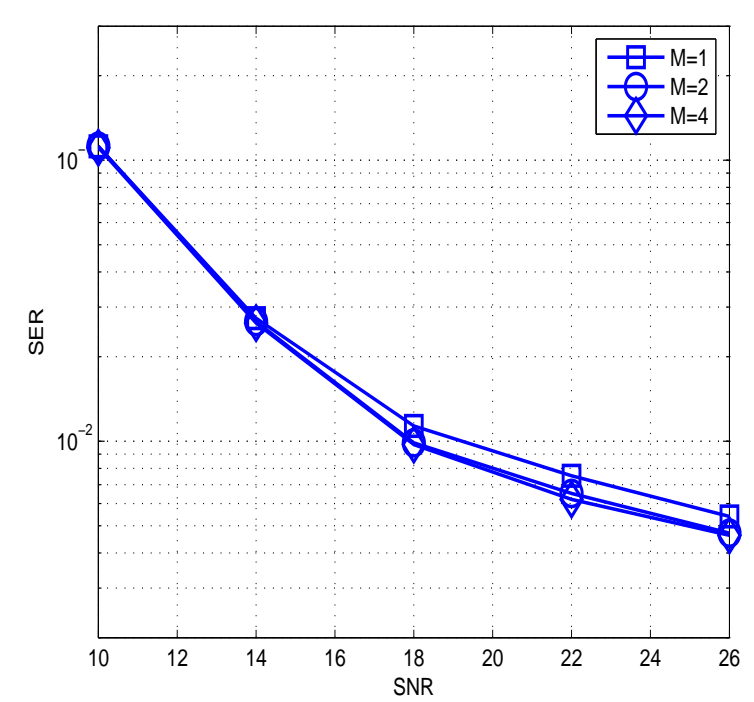

Fig. 4. Symbol error rate as a function of SNR for various values of $M$ for an $8 \mathrm{~K}$ DVB test channel: conventional system $(M=1)$, and partial FFT demodulation with varying number of intervals $M$.

[6] C.-Y. Hsu and W.-R. Wu, "Low-Complexity ICI mitigation methods for high-mobility SISO/MIMO-OFDM systems," IEEE Transactions on Vehicular Technology, vol. 58, no. 6.

[7] Y. Mostofi, D. Cox, and A. Bahai, "ICI mitigation for mobile OFDM receivers," in Communications, 2003. ICC '03. IEEE International Conference on, vol. 5, May 2003, pp. 33513355.

[8] A. B. Salberg and A. Swami, "Doppler and frequency offset synchronization in wideband OFDM," IEEE Transactions on Wireless Communications, vol. 4, no. 6, Nov 2005.

[9] M. Stojanovic, "MIMO OFDM over underwater acoustic channels," in Proceedings of 43rd Asilomar Connference on Signals, Systems and Computers, Nov 2009.

[10] K. Tu, D. Fertonani, T. M. Duman, and P. Hursky, "Mitigation of inter-carrier interference in OFDM systems over underwater acoustic channels," in Proceedings of IEEE Oceans Conference, June 2009.

[11] P. Baracca, S. Tomasin, L. Vangelista, N. Benvenuto, and A. Morello, "Per sub-block equalization and channel estimation for next generation handheld DVB," in Ultra Modern Communications and Workshops, ICUMT, Oct 2009.

[12] S.-J. Hwang and P. Schniter, "Efficient communication over highly spread underwater acoustic channels," in WuWNet '07: Proceedings of the second workshop on Underwater networks, 2007, pp. 11-18.

[13] S. Tomasin, A. Gorokhov, H. Yang, and J.-P. Linnartz, "Iterative interference cancellation and channel estimation for mobile OFDM," IEEE Transactions on Wireless Communications, vol. 4, no. 1, Jan 2005. 